\section{Physiological and Psychological Response to Floral Scent}

\author{
Hyunju $\mathrm{Jo}^{1}$ and Susan Rodiek \\ Department of Architecture, Center for Health Systems \& Design, Texas \\ A\&M University, 3137 TAMU, College Station, TX 77843-3137
}

\author{
Eijiro Fujii \\ Graduate School of Horticulture, Chiba University, 648 Matsudo, Chiba, \\ 271-8510, Japan
}

Yoshifumi Miyazaki

Center for Environment, Health and Field Sciences, Chiba University, 6-2-1

Kashiwanoha, Kashiwa, Chiba, 277-0882, Japan

\author{
Bum-Jin Park \\ Department of Environment \& Forest Resources, Chungnam National \\ University, Daejeon, Chungnam, 305-764, Korea
}

\section{Seoung-Won Ann \\ Department of Horticulture, Kongju National University, Yesan, Chungnam, 340-800, Korea}

Additional index words. people-plant interactions, human issues in horticulture, fragrance, cerebral and autonomic nervous system activities, semantic differential scale, profile of mood states

\begin{abstract}
To better understand how fragrance may enhance human health, this study examined psychophysiological responses to Japanese plum blossom fragrance. Although previous studies used essential oils or fragrance components, the present study measured the effects of floral scent naturally diffused by the plant itself to simulate the way we generally experience natural scent in everyday life. Subjects were Japanese males $(n=$ 26), and the data collected included cerebral and autonomic nervous system activities, semantic differential (SD) scale, and profile of mood states (POMS). Exposure to the fragrance significantly activated the sympathetic nervous system and the cerebral areas related to movement, speech, and memory. SD scale and POMS results showed the fragrance evoked cheerful, exciting, and active images and changed mood states by enhancing vigor while suppressing feelings of depression. These findings indicate that contact with a floral scent such as plum blossom fragrance can improve mood states and may foster the brain functions of memory, speech, and movement, potentially leading to improvements in emotional health, depression, and memory disorders.
\end{abstract}

Contact with plants has been found to be associated with health benefits, including improvements in physical, cognitive, psychological, and social functioning (Simson and Straus, 1998). The people-plant relationship has been explored in terms of theoretical concepts such as learning, evolution, and overload/arousal (Kaplan and Kaplan, 1989). For example, the evolution theory maintains that because we evolved in environments consisting primarily of plants, we tend to have positive psychological and physiological responses to them (Frumkin, 2001; Ulrich, 1983). Increasingly, empirical studies demonstrate that people tangibly benefit from contact with plants

\footnotetext{
Received for publication 19 Apr. 2012. Accepted for publication 7 Nov. 2012.

We appreciate the valuable contributions of the students at Chiba University in Japan, who were willing to participate in the experiment.

${ }^{1}$ To whom reprint requests should be addressed; e-mail hjjo1011@gmail.com.
}

and other nature elements using different kinds of measures in different populations (Kuroko and Fujii, 2002; Rodiek, 2002; Ulrich 1981, active involvement with nature such as gardening or viewing/experiencing a natural setting can have a positive impact on physical and mental health. These findings can be applied to the design of improved environments; they can also be used in therapeutic horticulture to create more effective healing experiences (Cooper Marcus and Barnes, 1999; Gonzalez et al., 2010).

Although there is empirical evidence of the restorative effects of nature and plants, a majority of studies have focused on either visual perception of nature (Chang and Chen, 2005; Parsons et al., 1998; Ulrich et al., 1991) or else active involvement with plants (Mcbey, 1985). However, therapeutic horticulture professionals have documented the importance of the non-visual sensory perceptions such as scent, sound, touch, and taste. Some have suggested that a sensory or memory garden 1984). These studies show that passive or should stimulate as many senses as possible by using natural elements such as sunlight, water, breezes, fragrance, and views of wildlife (Wagenfeld, 2009). Multisensory stimulation has been found to enhance the healing effects of nature, especially for specific users such as children, hospital patients, and persons with dementia (Chapman et al., 2005; Said and Abu Bakar, 2007).

In a nature setting, people may experience plant scents consciously or unconsciously. Gardens may be designed to take advantage of attractive floral scents, which may create feelings leading to specific kinds of therapeutic experience. Floral scents can often trigger memories of particular times, events, places, or feelings (Haas and McCartney, 1996). Studies of the brain have shown that the olfactory sense is closely related to the limbic system, which in turn is responsible for the function of instinct. The olfactory sense is also intimately related to mood and feelings and, unlike the other senses, is linked to long-term memory (Brennan et al., 1990; Engen, 1982; Herz et al., 2004; Onoda, 2000; Zatorre et al., 1992). The aspects of the olfactory sense relating to emotion and memory suggest the possible therapeutic benefits of floral scent. As a potential natural healing element, floral scent may provide substantial mental or emotional benefits such as relieving anxiety or depression and maintaining memory abilities for individuals with Alzheimer's disease or other memory disorders (Brawley, 2004; Cohen-Mansfield and Werner, 1999).

By measuring cerebral and autonomic nervous system responses, studies have demonstrated the physiological responses resulting from fragrances; for example, lavender fragrance increased beta power in electroencephalographic activity, suggesting increased drowsiness and also increased parasympathetic nervous activity in the direction associated with relaxation. Rosemary fragrance, on the other hand, decreased alpha and beta power, suggesting increased alertness; it also stimulated sympathetic nervous activity associated with excitement (Diego et al., 1998; Saeki and Shiohara, 2001). However, a majority of these studies has used essential oils or fragrance components instead of actual plant materials. Few previous studies have examined the effect of the scent that is naturally diffused by the plant, which is generally how we experience scent in everyday life or in horticultural activities. Kweon et al. (2003) compared the brain responses between people who were tearing peppermint leaves compared with people who were tearing paper and found those handling peppermint showed more vigor and brain stimulation than those handling paper. Interestingly, by comparing brain responses with intuitive verbal responses to the same scent, Jo et al. (2007) found that familiar plant scents such as shiso (Perilla frutescens) and Japanese pepper tree leaves (Zanthoxylum piperitum), often used as foods or medicines, are typically thought of as stimulating, whereas they actually have a relaxing effect on the prefrontal area in the cerebrum involved in judgment and feeling 
function. These studies suggest that the natural scent diffused by plants can have positive physical and mental benefits that may be useful in horticultural therapy.

The present study measured the immediate effects of natural floral scents on the cerebrum and autonomic nervous system as well as on psychological evaluations of mood and stimuli using the scent diffused by the flower itself. The findings presented here may lead to better understanding of how fragrance affects people in indoor and outdoor gardens, therapeutic environments, or during horticultural activities.

\section{Materials and Methods}

Participants and experimental setting. The participants were 26 Japanese male students in their mid-20s (mean age \pm SD, $24 \pm 1.8$ years) recruited from landscape and horticulture programs at Chiba University in Japan. This limits the generalizability of the study but had the advantage of reducing differences of olfactory response resulting from gender, age, and culture. Undergraduate and graduate volunteers were recruited by a flyer, and informed consent was obtained according to the regulations of the Human Investigation Committee of Chiba University. Interviews were used to screen those with relevant past or current physical and olfactory disorders such as insomnia or sinus infection with the potential to influence the physiological results. The experiment was conducted in a screened room at Chiba University, where subjects would be exposed to the fewest external influences and go through the test under the same conditions. In the middle of a shielded room $\left(59.4 \mathrm{~m}^{2}\right)$, with a white ceiling and walls, a chair was set for the subject. The biological measurement devices were placed behind the chair to reduce visual influences. A $77^{\circ} \mathrm{F}$ temperature and $60 \%$ relative humidity were maintained throughout the experiment, because the concentration of scent alters with changes in temperature and humidity (Bocca and Battiston, 1964).

Scent stimulus. The present study used the scent of Japanese plum blossoms (ume, or Prunus mume). The Japanese plum tree has traditionally been used as a garden plant and bonsai material, because of the beautiful flowers and fragrance of the blossoms (Horiuchi, 1996). For this study, the fragrance was obtained from the fresh petals collected a few hours before the experiment. Four grams of petals $(\approx 36$ petals, considered to emit an appropriate concentration of scent) and odorless air were put in a polypropylene bag. The scent was then allowed to concentrate naturally within the bag. The same type of odorless air was placed in a bag without plum blossom petals as a comparison for the plum blossom-scented air. To present the same concentration and intensity of scent to all subjects, to the extent possible, the scent and odorless air were presented using the process illustrated in Figure 1. Because the olfactory sense has a wide range of individual differences, the perceived intensity of the fragrance was evaluated ranging from odorless to intolerable (range of intensity: 0 to $4=$ odorless to faint; 4 to $8=$ faint to weak; 8 to $12=$ weak to strong; 12 to $16=$ strong to very strong; 16 to $20=$ very strong to intolerable). As a result of this method of evaluation, the plum blossom fragrance was reported as having an intensity between weak and strong (mean \pm $\mathrm{SD}, 9.34 \pm 3.5$ ), whereas the odorless air was evaluated as almost odorless (mean \pm SD, $1.23 \pm 1.58)$.

Measures. The study used a combination of biological measures and verbal responses. While each subject was exposed to the fragrance, cerebral and autonomic nervous system activities were recorded. After taking biological measurements, verbal evaluations were used to determine the subject's mood, mental images, and the emotional qualities evoked by exposure to either the plum blossom fragrance or the odorless air.

Cerebral activity. Changes in cerebral activity resulting from the scent stimuli were measured by multichannel near-infrared spectroscopy (NIRS; OMM-2001; Shimadzu Co., Ltd., Japan). Unlike contingent negative variation) or electroencephalogram, which use minute magnetic fields occurring from ion electronic charges in the cerebral blood, NIRS directly monitors regional relative changes of hemoglobin concentration in the cerebral blood flow (Villringer and Dirnafl, 1995). This method requires only compact experimental systems and is less restrictive, allowing the subject to move or be active in his or her seat, unlike functional magnetic resonance imaging) and positron emission tomography testing, which require subjects to remain in a supine position (Nakamura, 1996; Okamoto et al., 2004). Measurement was limited to the right brain hemisphere as the seat of activities related to emotions and image creation (Silberman and Weingartner, 1986; Tucker, 1981; Fig. 2A). A total of 47 measurement locations, referred to as channels (e.g., ch10, ch17, ch24), were located in the frontal, parietal, temporal, and occipital lobes (Fig. 2B). These brain locations correspond with feeling, judgment, premotor, motor, somatosensory, cognition, visual, auditory, and memory functions and indicate how the fragrance influenced subjects' memory and emotions (Caplan, 1993; Shepherd, 1901; Fig. 2C).

Autonomic nervous system activity. The autonomic nervous system is related to functions that are necessary for life such as respiration, heart rate, and blood pressure. Autonomic nervous system response indicates shifts in psychological factors such as emotion, motivation, attention, and preference as well as physical movement (Harmon and Beer, 2009). There are two main parts: the sympathetic nervous system (SNS) and the parasympathetic nervous system (PNS). In general, the SNS is activated by excitement and tension, which are accompanied by increased heart rate and blood pressure. The PNS is activated by relaxation and is associated with slower heart rate and decreased blood pressure (Robertson, 2004). For this study, a finometer device, which clips on the middle finger of the left hand (Fig. 3), was used to measure pulse rate and arterial blood pressure. The device can sequentially measure blood pressure and pulse rate during exposure to the scent stimuli and is less burdensome to the subjects as a result of the simplicity in wearing the device (Schutte et al., 2004). Three sensors attached to the chest (right collarbone; right and left ribs) were used to detect heart rate variability (HRV), as measured by an ambulatory

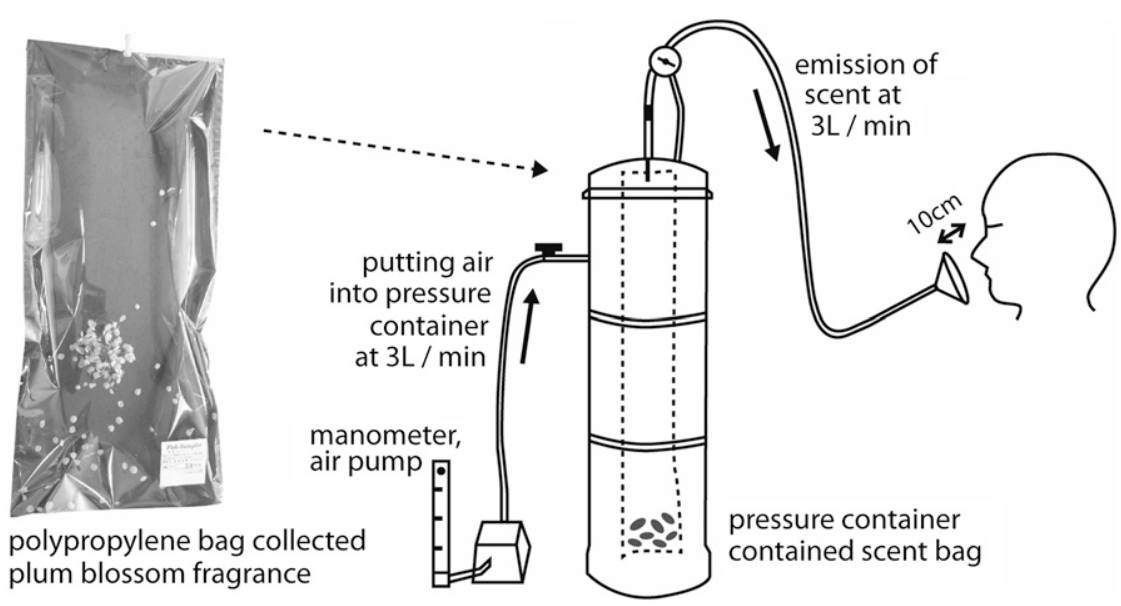

Fig. 1. Preparation and presentation process of plum blossom fragrance and odorless air. The scent stimuli were prepared as follows: (A) plum blossom fragrance: $4 \mathrm{~g}$ of plum blossom petals and odorless air were put into a polypropylene bag. The scent was then allowed to concentrate naturally within the bag; and (B) odorless air: into the same type of polypropylene bag, only odorless air was introduced as a comparison for the plum blossom-scented air. To present the same concentration and intensity of scent stimuli to all subjects, they were presented as follows: constant speed of the air $\left(3 \mathrm{~L} \cdot \mathrm{min}^{-1}\right)$ was put into a pressure container that contained the plum blossom fragrance bag or odorless air bag by an air pump with a manometer attached, emitting a consistent volume of plum blossom fragrance or odorless air. All participants were asked to inhale the scent at a distance of $10 \mathrm{~cm}$ from their nose. 

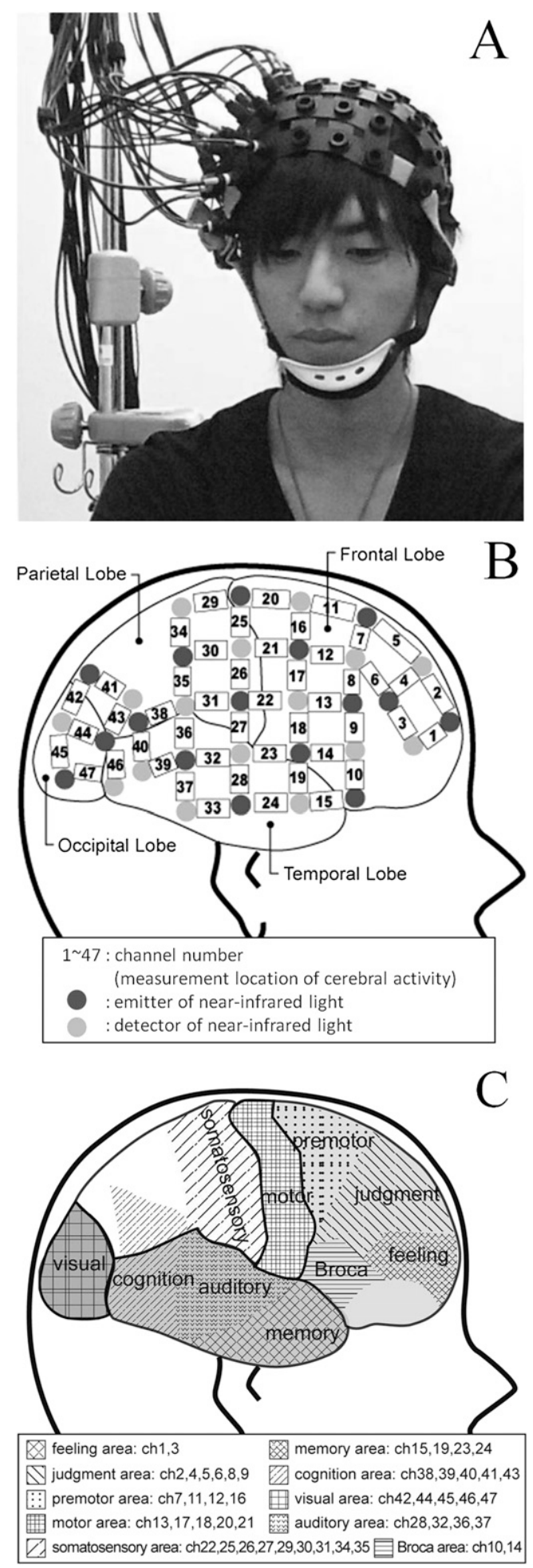

Fig. 2. Measurement of cerebral activity using multichannel near-infrared spectroscopy (NIRS). (A) The photograph shows the measurement of cerebral activity in the right brain hemisphere by the NIRS device. (B) The cerebral activity was detected in a total of 47 areas in cerebral cortex using electrodes (emitter and detector) of near-infrared light. The channel is the area between emitter and detector and indicates the location of cerebral activity. The 47 channels were located in the frontal, parietal, temporal, and occipital lobes. (C) According to the theory of localization of the brain function, 47 channels corresponded with feeling (ch1, 3), judgment (ch2, 4, 5, 6, 8, 9), premotor (ch7, 11, 12, 16), motor (ch13, 17, 18, 20, 21), somatosensory (ch22, 25, 26, 27, 29, 30, 31, 34, 35), memory (ch15, 19, 23, 24, 33), cognition (ch38, 39, 40, 41, 43), visual (ch42, 44, 45, 46, 47), auditory (ch28, 32, 36, 37), and speech (Broca, ch10, 14) functions.

electrocardiogram (AC-301A; GMS Corp., Japan). This method registers beat-to-beat variation in the heart rhythm, which reflects subtle changes in a person's emotional state with great sensitivity (Kobayashi et al., 1999).

Mood and stimuli evaluations. To better understand how subjects responded to the

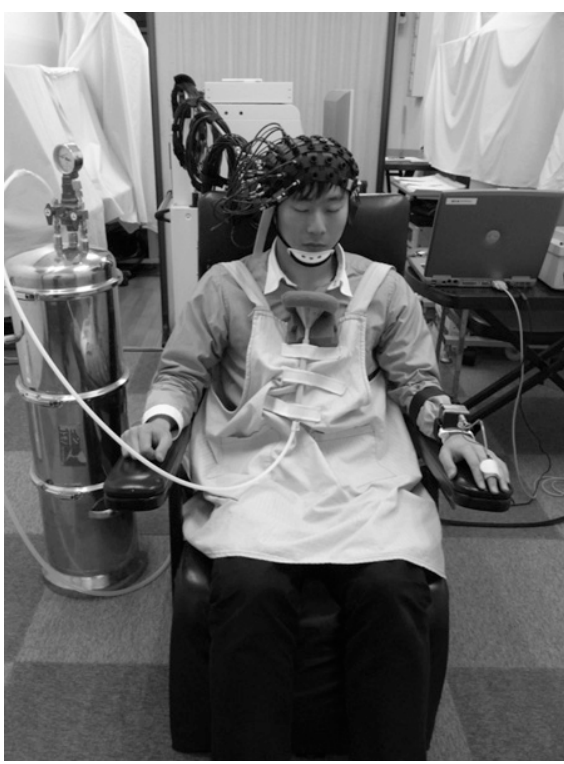

Fig. 3. (A-C) Measuring brain activity and other physiological responses during exposure to stimuli.

fragrance, two self-report questionnaires were introduced. First, mood was measured with the Profile of Mood States (POMS), which is a psychological rating scale that assesses short-term and distinct mood states (Pollock et al., 1979; Tsunetsugu et al., 2005). A brief version comprised of 30 items was used to reduce the time required for the measure (Profile of Mood States-Brief Form Japanese Version, Saccess Bell Co., Ltd., Japan). The items were rated on a 5-point scale ranging from "not at all" to "extremely" and included six types of mood states: tension-anxiety (T-A), depression-dejection (D), anger-hostility (A-H) as well as fatigue $(\mathrm{F})$, vigor $(\mathrm{V})$, and confusion $(\mathrm{C})$, as shown in Figure 7; in each case, a higher score indicates the subject is reporting higher levels of that mood state. The direct impressions evoked by the fragrance were examined using semantic differential (SD) methods, which have been found to be a reliable and valid way to quantify subjective feelings about external stimuli (Kang and Zhang, 2010; Lee et al., 2011; Snider and Osgood, 1969). In this study, 15 pairs of contrasting adjectives (e.g., artificial-natural, rough-delicate, woody-non-woody) were rated on a 7-point scale (Jo et al., 2007).

Experimental procedures. Figure $4 \mathrm{de}-$ scribes the overall experimental protocol, which took place over a 40-min period. After explaining the study details and protocol to each participant, the physiological measurement devices such as NIRS, HRV, and pulse rate/blood pressure and scent-emitting device were attached. The participant was then asked to relax fully during a rest period of $\approx 1$ to $2 \mathrm{~min}$ with eyes closed to adjust his mood to the experimental atmosphere. After the NIRS monitor confirmed that cerebral activity was stable, the plum blossom fragrance was presented for $2 \mathrm{~min}$ based on 
studies showing the olfactory adaptation phenomenon, in which sensory nervous activities decrease gradually when an odorant presents continually (Dalton, 2000). The presentation of scent stimuli was carried out without announcing that it was being done. During this time, physiological responses were measured, and questionnaires on the SD scale, POMS, and scent intensity were filled out. Although the written questionnaires took $\approx 5 \mathrm{~min}$ to complete, the fragranced air was presented only for the first 2 min. Then, the odorless air was presented for 2 min, also without being announced, and the same physiological and questionnaire responses were collected. The order was random with half the subjects receiving the fragrance first and half receiving the odorless air first.

Data analysis. The physiological changes during exposure to the fragrance were examined by comparing the means of each 30 -s interval starting with the last $30 \mathrm{~s}$ of the rest period before fragrance presentation (Fig. 5).
This was assumed to represent the most stable states of the brain and physiological activity during the rest period. A paired $t$ test (two-sided) was used to compare the physiological changes between rest and exposure periods. The cerebral activity analysis used changes in oxygenated hemoglobin (oxyHb) as the index of cerebral changes, where increased oxyHb is associated with increased cerebral activity. The changes were separately computed in 47 measurement locations of the brain. The HRV data were divided into 30 -s segments, and the beat-to-beat intervals were analyzed by the maximum entropy method. The program was set with the variance of the low-frequency (LF) band at 0.04 to $0.15 \mathrm{~Hz}$, and the variance of the highfrequency (HF) band at 0.15 to $0.4 \mathrm{~Hz}$. The HF data were used as an index of parasympathetic nervous system activity, and the LF/ $(\mathrm{LF}+\mathrm{HF})$ ratio was used as an index of sympathetic nervous activity (Lee et al., 2011). Because of the lack of normal distribution, the Wilcoxon signed-rank test (two-sided) was used to verify the differences in psychological effect assessed by the SD scale and POMS between the fragrance and odorless air. SD data were analyzed by comparisons of rating scores between the fragrance and odorless air in each contrasting item. POMS analysis was conducted as the rating scores of six types of mood states calculated as T-scores according to the calculation method standardized by POMS (Yokoyama et al., 1990). Statistical validity was established at $P<0.05$.

\section{Results}

Physiological responses. Figure 5 shows the time-series changes in oxyHb concentration, indicating cerebral activity during exposure to the fragrance and the odorless air in ch10 and ch24. Among 47 channels, the increases of oxyHb concentration by the fragrance exposure were significantly observed at three channels: ch10 (60 to $90 \mathrm{~s}, P=0.010)$, ch17 (30 to $60 \mathrm{~s}, P=0.006$ ), and ch24 (60 to $90 \mathrm{~s}, P=0.018 ; 90-120 \mathrm{~s}, P=0.024)$, unlike

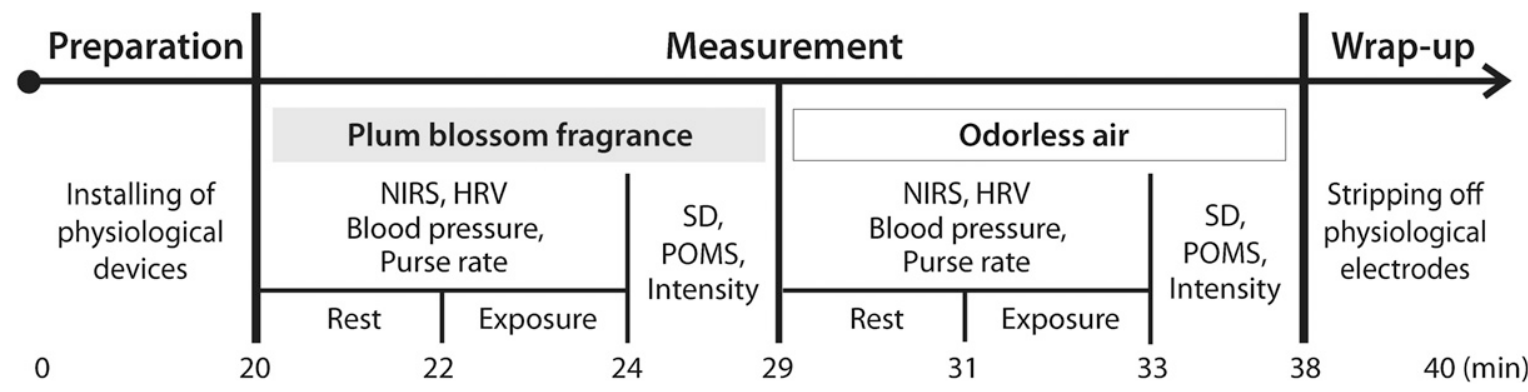

Fig. 4. Timeline (in minutes) of the physiological and psychological measurement during exposure to plum blossom fragrance and odorless air. The order was randomly reversed for half the subjects.

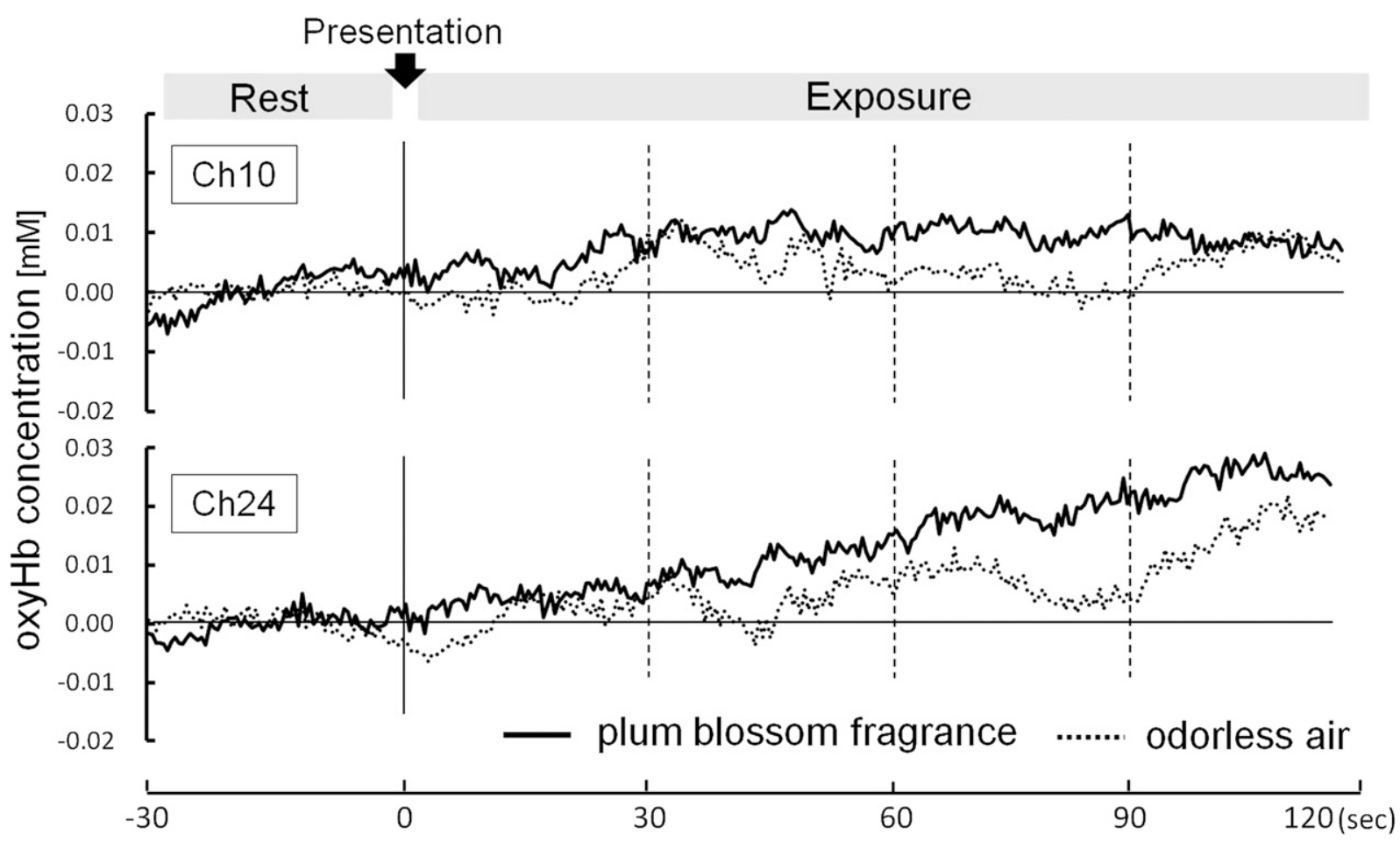

Fig. 5. Time-series changes in oxygenated hemoglobin (oxyHb) concentration (cerebral activity) during exposure to plum blossom fragrance and odorless air. 
the odorless air, which did not show a clear change in any channels (Table 1). Considering the localization of the functions in the brain (Fig. 3C), the activated channels were located in the motor area (ch17), Broca's area related to speech function (ch10), and the memory area (ch24). In the autonomic nervous system responses, blood pressure and pulse rate did not show any clear changes on exposure to the fragrance and odorless air (data not shown). However, HRV results showed significant physiological changes with exposure to the fragrance. Starting with exposure to the fragrance, the $\mathrm{LF} /(\mathrm{LF}+\mathrm{HF})$ ratio reflecting $\mathrm{SNS}$ activity was gradually increased compared with the rest period ( -30 to -1 s) with significant increases observed during 31 to $60 \mathrm{~s}$ and 61 to $90 \mathrm{~s}$ at $P<0.05$ (Fig. 6). Furthermore, cerebral and SNS responses both showed clear changes after $30 \mathrm{~s}$ of exposure to the fragrance and the effect lasted for 30 to $60 \mathrm{~s}$; these results indicate that somewhat consistent exposure to the fragrance is needed to obtain clearer physiological effects of the fragrance.

Mood and stimuli evaluations. Figure 7 shows the changes in mood states by exposure to the fragrance and odorless air; compared with odorless air, exposure to the fragrance resulted in a lower depressiondejection score $(\mathrm{D}, P<0.05)$ and a higher vigor score $(\mathrm{V}, P<0.01)$. These results indicate that the fragrance enhanced positive feelings such as vigor, whereas it reduced negative feelings such as depression. The SD results found that the fragrance evoked various positive impressions, showing significant differences in 13 adjectives (Fig. 8). The fragrance was evaluated as being natural, rural, woody, active, cheerful, stimulating, exciting, distinctive, pleasant, and dense $(P<$ $0.01)$; also firm, refreshing, and liked $(P<$ $0.05)$. It is considered that impressions such as cheerful, exciting, active, and pleasant are related to the higher vigor scores found in the POMS test.

\section{Discussion}

In recent decades, as the importance of contact with plants has become more apparent, researchers have increasingly demonstrated the health benefits of plants using a wide range of methods, measures, hypotheses, and populations. A great deal of research has focused on visual perception and horticultural activity, whereas there are relatively few studies on non-visual effects of plants through sensory perception such as scent, sound, or touch, although multisensory stimulation has been identified as an important way to enhance the healing effects of nature. The fragrance of plants, consciously or unconsciously experienced during contact with plants, can affect our mood and feelings directly because the olfactory sense is intimately connected to the limbic system, which is in charge of instinctive aspects such as feeling and appetite in the brain.

The present study demonstrated some of the potential benefits of exposure to floral
Table 1. Brain channels ${ }^{z}$ that observed significant changes in oxygenated hemoglobin (oxyHb) concentration among 47 channels during exposure to plum blossom fragrance and odorless air.

\begin{tabular}{|c|c|c|c|c|c|c|c|c|c|}
\hline & \multicolumn{4}{|c|}{ Plum blossom fragrance } & \multicolumn{4}{|c|}{ Odorless air } & \multirow[b]{2}{*}{ (s) } \\
\hline & $0-30$ & $30-60$ & $60-90$ & $90-120$ & $0-30$ & $30-60$ & $60-90$ & $90-120$ & \\
\hline$\overline{\operatorname{ch} 10}$ & $0.004 \mathrm{NS}$ & $0.009 \mathrm{NS}$ & $0.010^{*}$ & $0.009 \mathrm{NS}$ & $-0.001 \mathrm{NS}$ & $0.006 \mathrm{NS}$ & $0.002 \mathrm{NS}$ & $0.005 \mathrm{NS}$ & \\
\hline $\operatorname{ch} 17$ & $0.002 \mathrm{NS}$ & $0.006^{*}$ & $0.003 \mathrm{NS}$ & $-0.002 \mathrm{NS}$ & $-0.007 \mathrm{NS}$ & $0.008 \mathrm{NS}$ & $-0.002 \mathrm{NS}$ & $-0.010 \mathrm{NS}$ & \\
\hline $\operatorname{ch} 24$ & $0.004 \mathrm{NS}$ & $0.010 \mathrm{NS}$ & $0.018 *$ & $0.024 *$ & $-0.000 \mathrm{NS}$ & $0.003 \mathrm{NS}$ & $0.007 \mathrm{NS}$ & $0.013 \mathrm{NS}$ & $(\mathrm{mm})$ \\
\hline
\end{tabular}

zThe brain channel indicates the measurement location where near-infrared spectroscopy electrodes were placed. They were placed on 47 total areas in the right brain hemisphere. The means were compared between the rest period ( -30 to $-1 \mathrm{~s}$ ) and the exposure period (every $30 \mathrm{~s}$ ) to the fragrance and to the odorless air. Significant changes in oxyHb concentration were verified by paired $t$ test (two-sided).

* Significant increase in oxyHb concentration at $P<0.05$. NS $=$ nonsignificant difference.

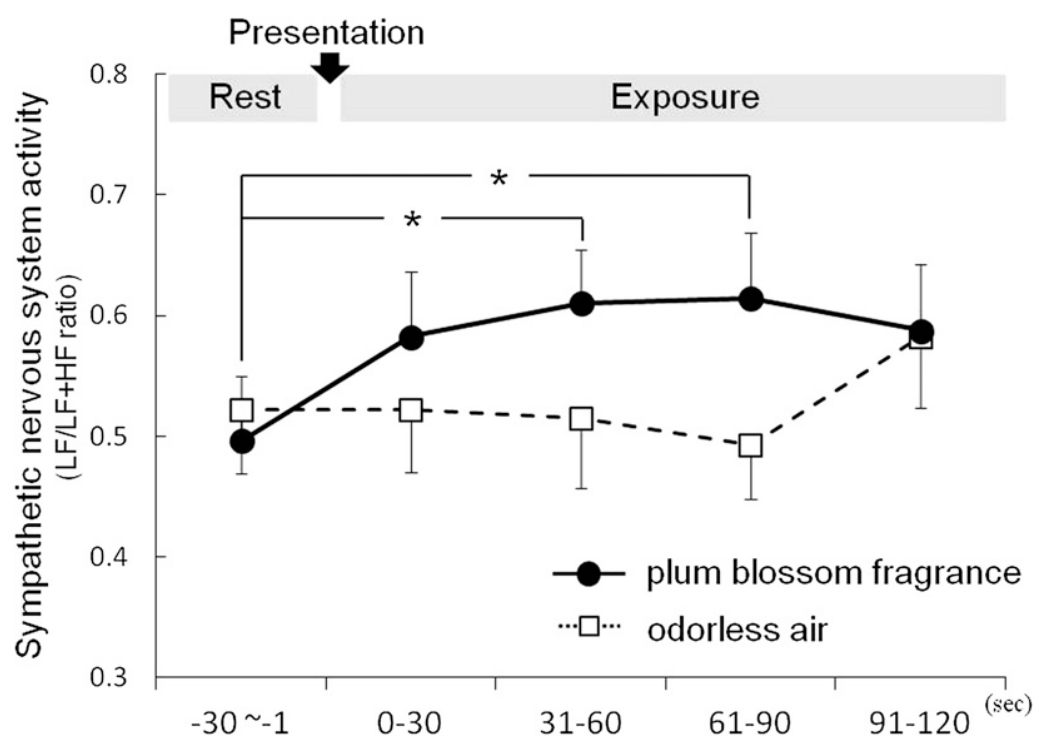

Fig. 6. Time-series changes in sympathetic nervous system activity during exposure to plum blossom fragrance and odorless air. Paired $t$ test (two-sided) comparison of the means between the rest period $(-30$ to $-1 \mathrm{~s})$ and the exposure period (every $30 \mathrm{~s})$ to the fragrance and odorless air. * Significance at $P<$ 0.05 . Mean \pm SE.

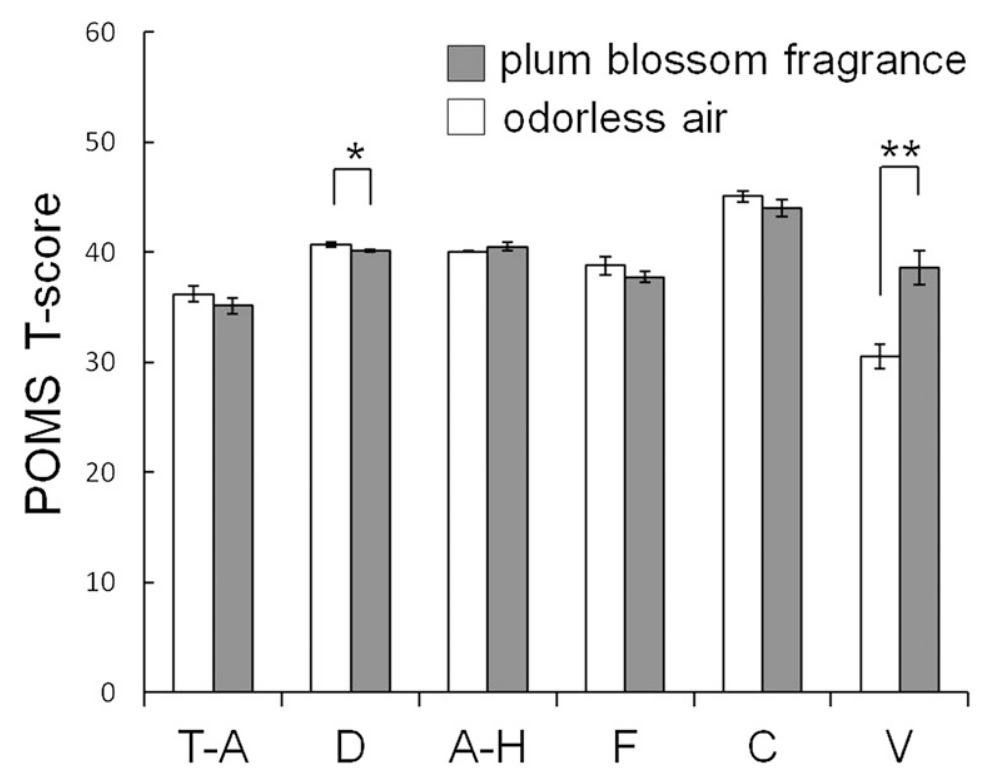

Fig. 7. T-score for the Profile of Mood States (POMS) with plum blossom fragrance and odorless air. T-A = tension-anxiety; $\mathrm{D}=$ depression-dejection; $\mathrm{A}-\mathrm{H}=$ anger-hostility; $\mathrm{F}=$ fatigue; $\mathrm{C}=$ confusion; $\mathrm{V}=$ vigor. Comparison of means between the plum blossom fragrance and odorless air; significant differences were verified by Wilcoxon signed-rank test (two-sided). *, ** Significance at $P<0.05$ or $<0.01$, respectively. Mean $\pm \mathrm{SE}$. 


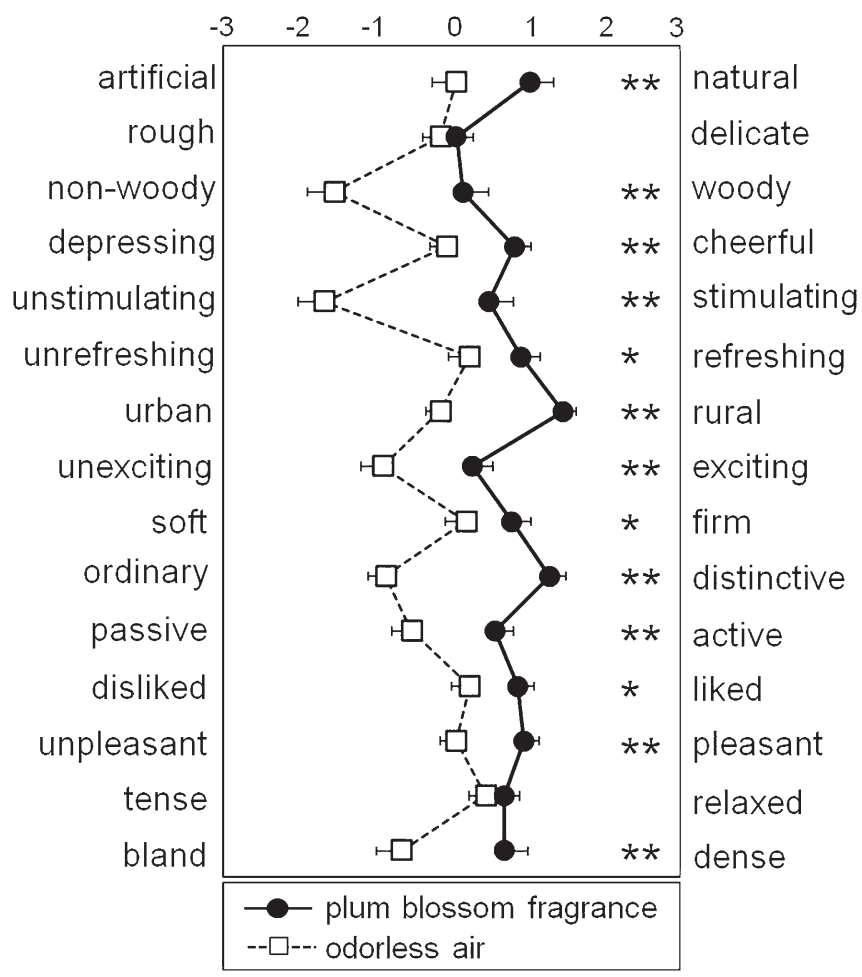

Fig. 8. Mean score of semantic differential (SD) scale with fragrance and odorless air. The SD scale was used to evaluate the direct impressions evoked by plum blossom fragrance and odorless air using 15 pairs of contrasting adjectives on scales ranging from -3 to 3 . The mean scores indicated the impressions of fragrance and odorless air, and significant differences were verified by Wilcoxon signed-rank test (two-sided). ${ }^{*}, *$ Significance at $P<0.05$ or $<0.01$, respectively; Mean \pm SE.

scent naturally diffused by flowers and verified multiple physiological and psychological responses using biological and verbal measures. Physiologically, the fragrance activated the cerebral areas in charge of movement, speech, and memory functions and stimulated the SNS activity in ways related to excitement. These results indicate that experiencing a floral scent such as plum blossom potentially can foster brain functions such as movement, speech, and memory and evokes a physiological effect similar to excitement. The finding of cerebral function activation is comparable with the results of previous research, in which the cerebral area in charge of speech was activated by exposure to pine scent (Jo et al., 2010). This helps to support earlier findings that involvement with plants through horticultural therapy tended to promote speech, communication, and thereby social interaction of participants with acquired aphasia and helped prevent deterioration of the mental abilities of individuals with Alzheimer's disease in longterm care (D'Andrea et al., 2007; Sarno and Chambers, 1997).

Psychologically, the plum blossom fragrance enhanced vigor while reducing depressed feelings and evoked cheerful, exciting, and active images. This helps explain the common experience that plant fragrances have a positive effect on our mood and emotions. It also supports the findings of previous studies on the benefits of plants such as the use of scented plants in horticultural activity or improvements found in mental and physical health through experiencing fragrance (Cooper Marcus and Barnes, 1999; Gonzalez et al., 2010; Wichrowski et al., 2005).

Limitations of the study. To reduce confounding variables, this experiment controlled the gender, age, and nationality of participants as well as environmental conditions such as noise, temperature, and humidity (Bocca and Battiston, 1964; Brand and Millot, 2001). Accordingly, the effects found in this study might not be generalizable to certain populations or different environmental conditions. This study also did not find the distinct benefits of lowered blood pressure and pulse rate that have been found in previous studies (e.g., Bensafi et al., 2002). This might have been because previous studies typically used longer exposures and stronger fragrances derived from aromatic oils rather than natural plant scents. Further studies are needed to test outcomes with a wider range of participants and methodologies as well as using a longer presentation time, collecting data on the response time of the cerebral region and possibly on the subject's thoughts and feelings during exposure to the fragrance. It would also be valuable to verify the effect of scents while simultaneously viewing nature elements, like in the situations we experience in everyday life.

\section{Conclusion}

This study's findings on the physiological and psychological effects of floral scent support common experiential knowledge of the effects of fragrance on humans and provide evidence that benefits of contact with plants are derived from not only through visual perception, but also through other sensory means such as olfactory perception. This strongly suggests that therapeutic use of plants should target multiple senses for optimal impact on physical, mental, and psychological health conditions.

\section{Literature Cited}

Bensafi, M., C. Rouby, V. Farget, B. Bertrand, M. Vigouroux, and A. Holley. 2002. Autonomic nervous system responses to odours: The role of pleasantness and arousal. Chem. Senses 27:703-709.

Bocca, E. and M.N. Battiston. 1964. Odour perception and environment conditions. Acta Otolaryngol. 57:391-400.

Brand, G. and J. Millot. 2001. Sex differences in human olfaction: Between evidence and enigma. J. Exp. Psychol. 54:259-270.

Brawley, E.C. 2004. Gardens of memories. Alzheimer's Care Quarterly 5:154-164.

Brennan, P., H. Kaba, and E.B. Keverne. 1990. Olfactory recognition: A simple memory system. Science 250:1223-1226.

Caplan, L.R. 1993. Brain-stem localization and function. Springer, New York, NY.

Chang, C. and P. Chen. 2005. Human response to window views and indoor plants in the workplace. HortScience 40:1354-1359.

Chapman, N.I., T. Hazen, and E. Noell-Waggoner. 2005. Encouraging development and use of gardens by caregivers of people with dementia. Alzheimer's Care Quarterly 6:349357.

Cohen-Mansfield, J. and P. Werner. 1999. Outdoor wandering parks for persons with dementia: A survey of characteristics and use. Alzheimer Dis. Assoc. Disord. 13:109-117.

Cooper Marcus, C. and M. Barnes. 1999. Healing gardens: Therapeutic benefits and design recommendations. John Wiley \& Sons, New York, NY.

D'Andrea, S.J., M. Batavia, and N. Sasson. 2007. Effect of horticultural therapy on preventing the decline of mental abilities of patients with Alzheimer's type dementia. J. Ther. Hort. 8: 9-13.

Dalton, P. 2000. Psychophysical and behavioral characteristics of olfactory adaptation. Chem. Senses 25:487-492.

Diego, M.A., N.A. Jones, T. Field, M. HernandezReif, S. Schanberg, C. Kuhn, V. McAdam, R. Galamaga, and M. Galamaga. 1998. Aromatherapy positively affects mood, EEG patterns of alertness and math computations. Intl. J. Neurosci. 96:217-224.

Engen, T. 1982. The perception of odors. Academic Press, New York, NY.

Frumkin, H. 2001. Beyond toxicity: Human health and the natural environment. Amer. J. Prev. Med. 20:234-240.

Gonzalez, M.T., T. Hartig, G.G. Patil, E.W Martinsen, and M. Kirkevold. 2010. Therapeutic horticulture in clinical depression: A prospective study of components. J. Adv. Nurs. 66:2002-2013.

Haas, K.L. and R.S. McCartney. 1996. The therapeutic qualities of plants. J. Hort. Therapy 8:61-67.

Harmon, E. and J.S. Beer. 2009. Methods in social neuroscience. Guilford Press, New York, NY.

Herz, R.S., J. Eliassen, S. Beland, and T. Souza. 2004. Neuroimaging evidence for the emotional 
potency of odor-evoked memory. Neuropsychologia 42:371-378.

Horiuchi, S. 1996. Ume of Japan and ume of world. Yokendo Press, Tokyo, Japan.

Jo, H., E. Fujii, and T. Cho. 2010. An experimental study of physiological and psychological effects of pine scent. J. Kr. I. Landsc. Arch. 38:1-10.

Jo, H., J. Lee, and E. Fujii. 2007. Physiological and psychological effects of Oriental herbs scents based on cerebral blood flow and semantic differential method. Environ. Inf. Sci. 21:207-212.

Kang, J. and M. Zhang. 2010. Semantic differential analysis of the soundscape in urban open public spaces. Build. Environ. 45:150-157.

Kaplan, R. and S. Kaplan. 1989. The experience of nature: A psychological perspective. Cambridge University Press, New York, NY.

Kobayashi, H., K. Ishibashi, and H. Noguchi. 1999. Heart rate variability: An index for monitoring and analyzing human autonomic activities. J. Physiol. Anthropol. Appl. Human Sci. 18:53-59.

Kuroko, N. and E. Fujii. 2002. An experimental study of noise stress recovery by inspecting green spaces using electroencephalogram, heart rate and subjective evaluation. J. Jpn. I. Landsc. Arch. 65:697-700.

Kweon, H., F. Asano, Y. Iwasaki, and S. Yamamoto. 2003. Effect of tearing with aroma plant (Mentha piperita var. vulgalis) and paper on the cerebral oxygenation. Hort. Landsc. Study 6:51-55.

Lee, J., B. Park, Y. Tsunetsugu, T. Ohira, T. Kagawa, and Y. Miyazaki. 2011. Effect of forest bathing on physiological and psychological responses in young Japanese male subjects. Public Health 125:93-100.

Mcbey, M. 1985. The therapeutic aspects of gardens and gardening: An aspect of total patient care. J. Adv. Nurs. 10:591-595.

Nakamura, N. 1996. Near-infared spectrophotometry and the application. Jpn. J. Physiol. Anthropol. 1:47-50.
Okamoto, M., H. Dan, K. Shimizu, K. Takeo, T. Amita, I. Oda, I. Konishi, K. Sakamoto, S. Isobe, T. Suzuki, K. Kohyama, and I. Dan. 2004. Multimodal assessment of cortisol activation during apple peeling by NIRS and fMRI. Neuroimage 21:1275-1288.

Onoda, H. 2000. Brain and odor: Neuroscience of olfactory. Kyoritsu Press, Tokyo, Japan.

Parsons, R., L.G. Tassinary, R.S. Ulrich, M.R. Heblam, and M. Grossman-Alexander. 1998. The view from the road: Implications for stress recovery and immunization. J. Environ. Psychol. 18:113-139.

Pollock, V., D.W. Cho, D. Reker, and J. Volavka. 1979. Profile of mood states: The factors and their physiological correlates. J. Nerv. Ment. Dis. 167:612-614.

Robertson, D.W. 2004. Primer on the autonomic nervous system. Academic Press, San Diego, CA

Rodiek, S. 2002. Influence of an outdoor garden on mood and stress in older persons. J. Ther. Hort. 13:13-21.

Saeki, Y. and M. Shiohara. 2001. Physiological effect of inhaling fragrances. Intl. J. Aromatherapy $11: 118-125$.

Said, I. and M.S. Abu Bakar. 2007. Affordances of ward and garden in the restorative process of hospitalized children. J. Hort. 18:18-31.

Sarno, M.T. and N. Chambers. 1997. A horticultural therapy program for individuals with acquired aphasia. Act. Adaptation Aging 22:81-93.

Schutte, A.E., H.W. Huisman, J.M. Rooyen, and N.T. Malan. 2004. Validation of the finameter device for measurement of blood pressure in black women. J. Hum. Hypertens. 18:79-84.

Shepherd, I.F. 1901. Localization of brain function. Kessinger Publishing, Whitefish, MT.

Silberman, E.K. and H. Weingartner. 1986. Hemispheric lateralization of functions related to emotion. Brain Cogn. 5:322-353.

Simson, S. and M.C. Straus. 1998. Horticulture as therapy: Principles and practice. The Haworth Press, New York, NY.
Snider, J.G. and C.E. Osgood. 1969. Semantic differential technique: A sourcebook. Aldine, Chicago, IL.

Tsunetsugu, Y., Y. Miyazaki, and H. Sato. 2005. Visual effects of interior design in actual-size living rooms on physiological responses. Build. Environ. 40:1341-1346.

Tucker, D.M. 1981. Lateral brain functions, emotion, and conceptualization. Psychol. Bull. 89:19-46.

Ulrich, R.S. 1981. Natural versus urban scenes: Some psycho-physiological effects. Environ. Behav. 13:523-556.

Ulrich, R.S. 1983. Aesthetic and affective responses to natural environment. Human Behav. \& Enviorn. Adv. in Theory \& Res. 6:85-125.

Ulrich, R.S. 1984. View through a window may influence recovery from surgery. Science 224: 420-421.

Ulrich, R.S., R.F. Simons, B.D. Losito, E. Fiorito, M.A. Miles, and M. Zelson. 1991. Stress recovery during exposure to natural and urban environments. J. Environ. Psychol. 11:201-230.

Villringer, A. and U. Dirnafl. 1995. Coupling of brain activity and cerebral blood flow: Basis of functional neuroimaging. Cerebrovasc. Brain Metab. Rev. 7:240-276.

Wagenfeld, A. 2009. It's more than seeing green: Exploring the senses through gardening. J. Ther. Hort. 19:46-52.

Wichrowski, M., J. Whiteson, F. Haas, A. Mola, and M.J. Rey. 2005. Effects of horticultural therapy on mood and heart rate in patients participating in an inpatient cardiopulmonary rehabilitation program. J. Cardiopulm. Rehabil. 25:270-274.

Yokoyama, K., S. Araki, N. Kawakami, and T. Takeshita. 1990. Production of the Japanese edition of profile of mood states (POMS): Assessment of reliability and validity [in Japanese]. Jap. J. Public Health 37:913-918.

Zatorre, R.J., M.J. Gotman, A.C. Evans, and E. Meyer. 1992. Functional localization and lateralization of human olfactory cortex. Nature 360:339-340. 